\title{
Physics of heat flow in the tails of needle crystals
}

\author{
Wim van Saarloos and John D. Weeks \\ AT\& T Bell Laboratories, Murray Hill, New Jersey 07974
}

(Received 2 October 1986)

\begin{abstract}
We show that the heat flow in the tail sections of needle-crystal solutions of dendritic growth becomes increasingly anisotropic. As a result, the dominant behavior in an outer expansion for the tails reduces to that of a very simple physical model which admits the exact Ivantsov solutions as well as a continuous family of solutions for nonzero capillary length. The model provides a useful testing ground for newly developed analytical and numerical methods for velocity selection.
\end{abstract}

Recent work ${ }^{1,2}$ has demonstrated that capillary forces can play a crucial role in the steady-state propagation of interfacial patterns. In the case of viscous fingering in a Hele-Shaw cell, the surface tension $\sigma$ enters as a singular perturbation in the equation for the finger width; it destroys the continuous family of solutions found for $\sigma=0$ and allows only a discrete set of finger widths, one of which can be identified with the unique value found in experiments. ${ }^{3-5}$

The existence of such a "solvability condition" is often understood in terms of ideas that were first applied in numerical studies of the Hele-Shaw equations, as well as to simple local models for dendrite growth. ${ }^{1,2,6}$ On relaxing the boundary condition at the tip of the finger (the "inner region") in the steady-state equation, a continuous family of physically acceptable solutions can be found in the tails (the "outer region"). However, only for particular parameter values is there a smooth joining of the "outer solution" to an "inner solution" which meets the proper boundary conditions at the tip; when this matching occurs, there exists a globally acceptable solution.

While there are a number of indications ${ }^{6-9}$ that a similar capillary-induced solvability condition could also apply to the steady propagation of dendrite tips, there are some important physical differences arising from the diffusive dynamics of heat flow. In this paper we focus on these differences and their possible consequences.

In the "matching picture," based essentially on the results of the local models, ${ }^{1,2}$ a continuous family of solutions can occur if the tails of the needles are very "forgiving," i.e., can join up to almost any possible tip behavior. Roughly speaking, in this case the behavior in the outer region (the tails) is analogous to that of a stable fixed point, which is approached by many nearby trajectories. ${ }^{10}$ With many "tails" to choose from, matching the tip and the tail solutions in the region of overlap is then generally possible.

Below we will analyze the steady-state equations for diffusion-controlled crystal growth and show nonperturbatively in the capillary length $d_{0}$ that the dominant behavior in an outer expansion for the tails has precisely the abovementioned properties. The argument is based on the fact that the equation for the outer region becomes exact everywhere in the limit of extremely anisotropic diffusion. This limit can be understood in very simple physical terms and helps to illustrate how a continuous family of solutions can arise; it should therefore serve as a useful testing ground for numerical and analytical tools.

For concreteness, we study steady-state propagation in two dimensions using the "symmetric model." 11 We imagine that anisotropic heat diffusion can occur, so that the temperature diffusion equation in a frame moving with the tip velocity $V$ becomes

$$
V \frac{\partial T}{\partial z}+D\left(\frac{\partial^{2} T}{\partial x^{2}}+\varepsilon^{2} \frac{\partial^{2} T}{\partial z^{2}}\right)=0 .
$$

The usual case of isotropic heat diffusion corresponds to $\varepsilon=1$. The boundary conditions at the interface, in dimensionless units, are

$$
\begin{aligned}
& \left(\mathbf{V}_{n}\right)_{x}=-D\left(\nabla T_{l}-\nabla T_{s}\right)_{x}, \\
& T_{i}=\Delta-d_{0} \kappa, \\
& \left(\mathbf{V}_{n}\right)_{z}=-\varepsilon^{2} D\left(\nabla T_{l}-\nabla T_{s}\right)_{z} .
\end{aligned}
$$

Here $\Delta$ is the dimensionless undercooling, $d_{0}$ the capillary length, $\boldsymbol{\kappa}$ the interface curvature, and $\mathbf{V}_{n}$ its normal velocity.

As in the isotropic case $\varepsilon=1$, Eqs. (1) and (2) can be written with the aid of Green's-function techniques ${ }^{11,12}$ as an integro-differential equation for the interface $z(x)$,

$$
\Delta-\gamma \kappa(x)=\frac{1}{\varepsilon \pi} \int_{-\infty}^{+\infty} d x_{1} \exp \left\{\varepsilon^{-2}\left[z\left(x_{1}\right)-z(x)\right]\right\} K_{0}\left(\varepsilon^{-2}\left\{\varepsilon^{2}\left(x-x_{1}\right)^{2}+\left[z(x)-z\left(x_{1}\right)\right]^{2}\right\}^{1 / 2}\right)
$$

Here all lengths are measured in units of $2 D / V$, the diffusion length at the tip, $K_{0}$ is the modified Bessel function of zeroth order, and $\gamma=d_{0} V / 2 D$ is the dimensionless capillary parameter.

Before analyzing the case $\gamma \neq 0$, we note that the Ivantsov solutions ${ }^{11,13}(\gamma=0)$ are independent of $\varepsilon$ : One can scale $\varepsilon$ out of the $\gamma=0$ equation by defining $\tilde{z}$ $=\varepsilon^{-1} z, \tilde{x}=\varepsilon^{-1} x$. Equation (3) then reduces to the integral equation for the isotropic case $(\varepsilon=1)$, and thus has Ivantsov solutions $\tilde{z}=-\tilde{x}^{2} / 2 p$, with the Péclet number $p$ given by the usual expression for the symmetric model. ${ }^{11,13}$ It follows that $z=-x^{2} / 2 p$ is also an Ivantsov solution of 
the original anisotropic equation.

At first glance, the above result may appear surprising, since small $\varepsilon$ suppresses heat flow in the $z$ direction. As a result, isotherms near the needle tip crowd close to one another [see Fig. 1(a)]. However, heat flow in the tails is increasingly in the $x$ direction, so the $z$ dependence of the temperature becomes dominanted by the convective $V \partial T /$ $\partial z$ term in Eq. (1). This is true for all $\varepsilon$, and it determines the entire Ivantsov needle shape.

To make this more precise, we note that according to (2), the relevant length scale for variations in the temperature field is the diffusion length $l=D / V_{n}=D / V \cos \theta$, where $\theta$ is the angle the normal makes with the $z$ direction. Thus $\partial T / \partial n=(\nabla T)_{n} \sim 1 / l$, so that $V \partial T / \partial z=V \cos \theta / l$. The derivative $D \partial^{2} T / \partial z^{2}$ has two contributions, a term of order $D(\cos \theta / l)^{2}$ from taking the second derivative in the normal direction, and a term resulting from the change in the angle $\theta$ with $z$ ( related to the curvature of the interface). It can be shown that the $\theta$ dependence of this term is of the same form as the first one, and thus we get

$$
R \equiv \frac{\varepsilon^{2} D \partial^{2} T / \partial z^{2}}{V \partial T / \partial z} \simeq \varepsilon^{2} g(p) \cos ^{2} \theta
$$

where $g(p) \sim p^{-1}$ for small $p$. Clearly, the ratio $R$ goes to zero in the tails $(\theta \rightarrow \pm \pi / 2)$, and so the third term in (1) becomes negligible in that region for any value of $\varepsilon$ and $p>0$. Note, however, that $R \rightarrow \infty$ if the zero Péclet limit is taken first, indicating that this limit must be taken with care.

This idea also helps analyze the case $\gamma \neq 0$. Consider a point in the tails for which $x$ is large enough so that $\varepsilon^{-1} z^{\prime} \gg 1$; i.e., $x \gg \varepsilon p$, where $z^{\prime}=d z / d x$. At these points, the dominant contributions to the integral in Eq. (3) come from these values of $\left|x_{1}\right|<|x|$ where the argument of the Bessel function $K_{0}$ is large; the integral from $x$
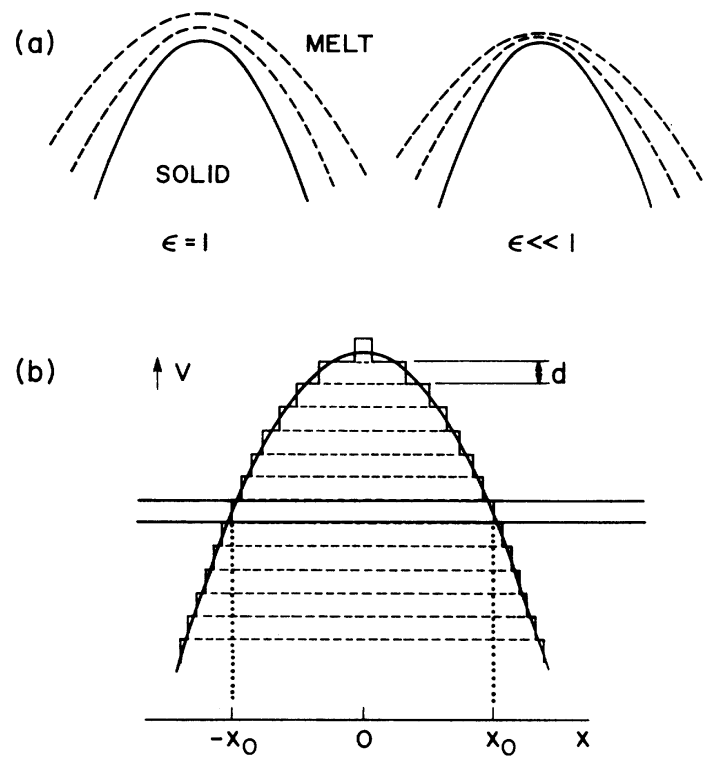

FIG. 1. (a) Isotherms near a dendrite tip for $\varepsilon=1$ and $\varepsilon \ll 1$. (b) To understand the $\varepsilon=0$ limit, the needle is imagined to consist of little layers of thickness $d$. The steady-state profile in the limit $d \rightarrow 0$ is the smooth interpolation of the pieces of interface in the separate layers.

$\leq x_{1}<\infty$ is negligible, since both the exponential factor and the Bessel function drop rapidly for $x_{1}>x$. Using the asymptotic expansion $K_{0}(w) \sim(\pi / 2 w)^{-1 / 2} e^{-w}$ and expanding the square root using the fact that $\varepsilon\left(x-x_{1}\right) /$ $\left[z\left(x_{1}\right)-z(x)\right] \ll 1$, we then get, for $x \gg \varepsilon p$, the "outer expansion" result

$$
\Delta-\gamma \kappa=\frac{1}{\sqrt{2 \pi}} \int_{-x}^{+x} d x_{1} \frac{\exp \left\{-\left(x-x_{1}\right)^{2} / 2\left[z(x)-z\left(x_{1}\right)\right]\right\}}{\left[z(x)-z\left(x_{1}\right)\right]^{1 / 2}}+\cdots, x \gg \varepsilon p .
$$

The right-hand side of this equation is correct to terms of order $\varepsilon / z^{\prime} \approx \varepsilon p / x$. The appearance of this ratio is not surprising, since the combination $\varepsilon^{2} \cos ^{2} \theta$ in (4) is of order $(\varepsilon p / x)^{2}$ in the tails. Because this ratio becomes small, the physics in the tails for any $\varepsilon$ is that of highly anisotropic heat flow. If we consider $\varepsilon$ small we can extend the range of $x$ where this outer expansion holds true, and for $\varepsilon=0$, Eq. (5) is exact for all $x$. This corresponds to a model system where heat exchange in the $z$ direction is completely suppressed. This limit allows us to interpret (5) physically; we call the model system with $\varepsilon=0$, defined by

$$
\begin{array}{r}
\Delta-\gamma \kappa=\int_{-x}^{+x} d x_{1} \frac{1}{\sqrt{2 \pi \tau}} e^{-\left(x-x_{1}\right)^{2} / 2 \tau} ; \\
\tau=z\left(x_{1}\right)-z(x), \text { all } x,
\end{array}
$$

the outer expansion model (OEM).

Consider a thin slice of this $\varepsilon=0$ system in the lab frame, as sketched in Fig. 1(b). If the instantaneous interface positions in this slice are $\pm x_{0}$, then the interface temperature is determined solely by the heat released earlier in this slice when the two interfaces were at positions $|x|<x_{0}$. The right-hand side of Eq. (6) expresses the interface temperature as the sum of all these contributions-indeed the integral is the well-known Green's function of the one-dimensional temperature equation, with $\tau$ the time it took the interface to move from $x_{1}$ to $x$. [In dimensional units, the steady-state interface must be at the same position $x_{1}$ in a slice a distance $V \tau$ higher, so that $V \tau=z\left(x_{1}\right)-z(x)$; in dimensionless units, this gives $\left.\tau=z\left(x_{1}\right)-z(x)\right]$.

Since the $\varepsilon \rightarrow 0$ limit is well defined, the $\varepsilon$-independent Ivantsov solution $z=-x^{2} / 2 p$ of (3) is also the Ivantsov solution of the OEM (6). Moreover, a family of solutions still exists in the OEM for $\gamma>0$. To understand this, let us imagine the system to consist of thin layers of thickness $d$ [Fig. 1(b)] and construct a solution with a given velocity $V$ by letting new solid slices nucleate at $x=0$ in successive layers every $\Delta t=d / V$ seconds. Once formed, each solid slice will grow out horizontally as long as the interface curvature $\kappa_{t}$ at the tip (obtained by smooth interpolation of the profile) is well defined in the limit $d \rightarrow 0$ (with $d / \Delta t=V$ fixed $),$ and provided that $\Delta-\gamma \kappa_{t}>0$ so that the melt is undercooled with respect to the tip temperature. 
Once started, the physics of each layer is just that of an interface in one dimension growing into an undercooled melt: The buildup of the heat in front of each slice will cause it to slow down for large times irrespective of the initial conditions (i.e., when each slice was part of the tip region) or of the presence of the coupling of the layers due to the $\gamma \kappa$ term. Thus, the OEM can "grow into the steady state." The tails do not constrain the growth in the tip region, but rather join smoothly onto the tip profile. As a result, the OEM has a continuous family of steady-state solutions for $\gamma>0$ due to the very mechanism we argued for earlier ${ }^{10}$ on physical grounds.

The condition for the existence of the limit $d \rightarrow 0$ at the tip, and hence for the existence of steady-state solutions, can be worked out explicitly. Writing $z=-x^{2} / 2 p_{\gamma}+\cdots$, with $p_{\gamma}$ the Péclet number for $\gamma>0$ and taking the limit $x \rightarrow 0$ in Eq. (6), we get

$$
\Delta-\frac{\gamma}{p_{\gamma}}=\sqrt{\pi p_{\gamma}} e^{p_{\gamma}} \operatorname{erfc}\left(\sqrt{p_{\gamma}}\right)
$$

This equation relates the dimensionless surface tension $\gamma$ to the Péclet number $p_{\gamma}$, and hence allows a continuous family of steady-state solutions, parametrized, e.g., by the tip radius of curvature. In fact, Eq. (7) is identical to the result found in the modified Ivantsov approximation, ${ }^{11}$ and so in spite of the fact that the heat flow in the tip region is unrealistic, ${ }^{14}$ the OEM still captures some essential features of the effect of capillary corrections on the tip.

Let us now return to the full problem with isotropic heat flow $(\varepsilon=1)$. Before discussing the implications for the matching picture, we consider the tip and tail region in more detail.

Equation (7) yields an upper bound for the values of $\gamma$ for which solutions can exist in the full model $(\varepsilon=1)$ without crystalline anisotropy. This is due to the fact that the isotherms become spaced closer together for decreasing $\varepsilon$ [see Fig. 1(a)], so that a solution with small $\varepsilon$ does not need to sharpen up as much in the tip region as an $\varepsilon=1$ solution, in order to accommodate the same temperature depression $d_{0} \kappa$ in $T_{i}$. Equation (7) is therefore useful in analyzing numerical solutions of Eq. (3), which are sometimes carried out using values of $\gamma$ above the upper bound (7).

The asymptotic behavior for $\varepsilon=1$ can also be studied quantitatively with the aid of Eq. (5). Since $z(x)$ approaches the Ivantsov solution $z=-x^{2} / 2 p$ for large $x$, we can write $z=-x^{2} / 2 p+\Delta z$ and linearize in $\Delta z$. After transforming to the variable $v=\left(x-x_{1}\right) /\left(x+x_{1}\right)$, we get $^{15}$

$$
-\gamma \kappa=\frac{p^{3 / 2}}{2 \pi^{1 / 2} x^{2}} \int_{0}^{\infty} d v \frac{1+v}{\sqrt{v}} e^{-p v}\left(p-\frac{1}{2 v}\right)\left[\Delta z\left(\frac{x(1-v)}{1+v}\right)-\Delta z(x)\right] .
$$

The $\kappa$ term on the left-hand side of this equation falls off as $1 / x^{3}$ for large $x$. Two cases have to be distinguished in discussing the leading behavior of $\Delta z$ : One possibility is that this term, when substituted in (8), gives rise to a contribution of order $x^{-3}$ on the right-hand side; alternatively, the dominant term in $\Delta z$ could give a vanishing contribution to the right-hand side of (8), in which case a subdominant term balances the $O\left(x^{-3}\right)$ term on the left-hand side. We consider the latter possibility first.

Because of the presence of the $p-1 / 2 v$ term, the integrand of (8) changes sign. Therefore, the integral can vanish when $p$ is not too small. Indeed, if we substitute $\Delta z=A x^{\beta}$, the integral on the right-hand side becomes proportional to $A f(p, \beta) x^{\beta-2}$, with

$$
f(p, \beta)=\int_{0}^{1} d v \frac{1+v}{\sqrt{v}}\left[e^{-p v}(p-1 / 2 v)+e^{-p / v}\left(p / v^{2}-1 / 2 v\right)\right]\left[\left(\frac{1-v}{1+v}\right)^{\beta}-1\right] .
$$

Here we have rewritten the integral from 1 to $\infty$ with the transformation $v \rightarrow 1 / v$. It is easy to see that the term between the first square brackets changes sign for $p>1 / 2$. Since the term between the second square brackets weights different parts of the interval $[0,1]$ differently depending on $\beta$, it is possible to make $f(p, \beta)$ vanish for some particular value of $\beta$ for any $p>\frac{1}{2}$. This implies that $\Delta z=A x^{\beta}$ for $p>\frac{1}{2}$, with the exponent $\beta$ determined by the requirement $f(p, \beta)=0$. As shown in the inset of Fig. $2, \beta$ approaches -1 for $p \rightarrow \frac{1}{2}$, and increases rapidly toward 1 for large $p$. We indeed find this asymptotic behavior in numerical solutions of the integral equations for $\varepsilon=0$ as well as for $\varepsilon=1$. Figure 2 shows the data for an undercooling $\Delta=0.76(p=1)$ on a log-log plot. For values of $x$ of about $10, \beta=\frac{1}{3}$ is found numerically; ${ }^{16}$ for larger $x$ the effective exponent continues to approach its predicted value of about 0.2. Note that the data for the full problem follow those of the OEM quite closely.

For $p<\frac{1}{2}, f(p, \beta)$ is always nonzero; as explained

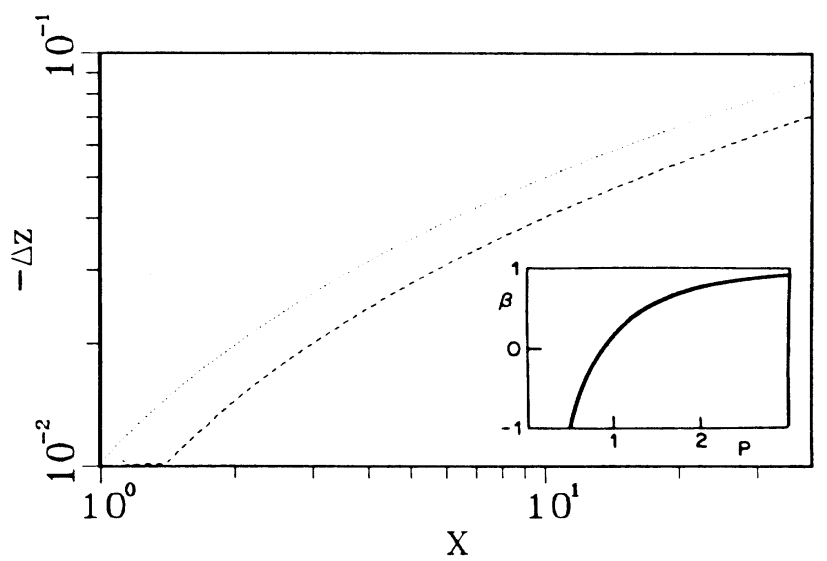

FIG. 2. $\Delta z$ vs $x$ on a $\log$ - $\log$ scale at $\Delta=0.76(p=1), \gamma=0.003$ for $\varepsilon=0$ (dashed line) and $\varepsilon=1$ (dotted line). Inset: $\beta(p)$ for $p>\frac{1}{2}$. 
above, the leading behavior of $\Delta z$ is now expected to give rise to a contribution of order $x^{-3}$ in (8). In a paper by Kotliar and the present authors, ${ }^{15}$ it will be shown that terms of this order arise from all parts of the profile, and that this implies that $\Delta z$ has to be integrable; the functional form of the behavior of $\Delta z$ for large $x$, however, is not fixed by the asymptotic analysis. In numerical solutions, it is found that $\Delta z \sim x^{-a}$ with $\alpha$ a noninteger exponent.

How relevant are the results of the OEM to the real system with $\varepsilon=1$ ? By construction, the OEM captures the essential physics of the full problem in the tails at distances greater than about a diffusion length $D / V$ from the tip, and thus provides evidence that there are many "flexible tails" available for matching in this region. In the simplest matching picture, this hints at the existence of a family of steady-state solutions.

However, at small Péclet numbers the diffusion length is much larger than the radius of curvature of the experimentally selected tip $\rho_{s}$, which scales ${ }^{11}$ as $\rho_{s} \sim \sqrt{d_{o} D / V}$. While the local models ${ }^{1,2}$ suggest that a matching condition in the tails governs the steady-state properties, this is not necessarily the case for the full nonlocal problem. The dynamically important region, before sidebranching becomes significant, clearly is of order $\rho_{s}$. If this physics reflects itself in the steady-state problem, then a likely scenario is that the selection can be thought of as arising from a nontrivial matching condition in the intermediate region of order $\rho_{s}$. A final matching to the "flexible tails" of the OEM, necessary to obtain a global solution, would in this view then be easy to achieve and have no implications for the solvability condition.

This picture seems self-consistent provided the deviations from the Ivantsov solution are very small in the tails. However, the present analysis has shown that there is a slow power-law approach to the Ivantsov solution in the tails, in contrast to the fast exponential approach found in the Laplace limit for the Hele-Shaw cell. This difference could affect the applicability of the solvability condition. Thus, there is a need for a more general analysis that considers explicitly both the intermediate region and the tails. It seems to us possible, at least for large Péclet numbers where the distinction between the two regions is much less clear cut, that the physics in the tails given by the OEM could dominate, and yield a family of solutions. If so, it will be important to study the three-dimensional problem in detail, since a given undercooling $\Delta$ corresponds to a much larger Péclet number in three dimensions than in two.

In mathematical studies of the full nonlocal problem, it is not clear how relevant the matching picture is, and most workers understand the solvability condition in terms of an analysis near a pole in the complex plane associated with the small argument logarithmic singularity of the modified Bessel function $K_{0}(w) .{ }^{9}$ From this perspective, the most serious mathematical objection to the OEM, which focuses on the large-argument behavior, is that the $\ln w$ singularity is repaced by a $w^{-1 / 2}$ singularity. However, an important assumption in the analysis, which is related to ideas from the matching picture, is that there is at most one solution of the integral equation. Again, it appears that a unified treatment is called for, which takes account of both the proper small argument singularity and the large argument behavior as in the OEM.

Quite independent of these considerations, the OEM can serve as a very useful testing ground for the newly developed analytical methods, and as a check on the numerical methods ${ }^{7,8}$ that have been applied to the full problem. It has already been argued ${ }^{10}$ that the method by which integral equations like (3) are investigated might fail to detect the existence of a family of solutions. Our numerical results for the OEM support this idea and will be reported in a future paper.
${ }^{1}$ E. Ben-Jacob, N. Goldenfeld, J. S. Langer, and G. Schön, Phys. Rev. Lett. 51, 1930 (1983); Phys. Rev. A 29, 330 (1984); E. Ben-Jacob, N. Goldenfeld, G. Kotliar, and J. S. Langer, Phys. Rev. Lett. 53, 2110 (1984).

${ }^{2}$ R. Brower, D. A. Kessler, J. Koplik, and H. Levine, Phys. Rev. Lett. 51, 1111 (1983); Phys. Rev. A 29, 1335 (1984); D. A. Kessler, J. Koplik, and H. Levine, ibid. 30, 2820 (1984); 30, 3161 (1984); 31, 1713 (1985).

${ }^{3}$ B. I. Shraiman, Phys. Rev. Lett. 56, 2028 (1986).

${ }^{4}$ D. C. Hong and J. S. Langer, Phys. Rev. Lett. 56, 2032 (1986).

${ }^{5} \mathrm{R}$. Combescot, T. Dombre, V. Hakim, Y. Pomeau, and A. Pumir, Phys. Rev. Lett. 56, 2036 (1986).

${ }^{6} \mathrm{See}$, for a general introduction and review, J. S. Langer, Institute for Theoretical Physics, University of California, Santa Barbara, Report No. 85-79, 1985 (unpublished); D. A. Kessler, J. Koplik, and H. Levine (unpublished).

${ }^{7}$ D. Meiron, Phys. Rev. A 33, 2704 (1986).

${ }^{8}$ D. A. Kessler, J. Koplik, and H. Levine, Phys. Rev. A 33, 3352 (1986).

${ }^{9}$ A. Barbieri, D. C. Hong, and J. S. Langer, Phys. Rev. A 35, 1802 (1987); M. Ben Amar and Y. Pomeau, Europhys. Lett.

\section{2, 307 (1986)}

${ }^{10} \mathrm{~A}$ local model with precisely such properties was given by W. van Saarloos and J. D. Weeks, Phys. Rev. Lett. 55, 1685 (1985). See also J. D. Weeks and W. van Saarloos, Phys. Rev. A (to be published).

"IJ. S. Langer, Rev. Mod. Phys. 52, 1 (1982).

${ }^{12}$ G. Nash, Naval Research Laboratory Reports No. 7679 and No. 7680, 1974 (unpublished).

${ }^{13}$ G. P. Ivantsov, Dokl. Akad. Nauk. SSSR 58, 567 (1947) [Sov. Phys. Dokl. 32, 391 (1947)].

${ }^{14}$ While the OEM captures the essential features of steady-state needles in the tails, dynamical properties are unrealistic since the Mullins-Sekerka instability is completely suppressed.

${ }^{15}$ In a paper by Kotliar and the present authors [Wim van Saarloos, John D. Weeks, and Gabriel Kotliar, this issue, Phys. Rev. A 35, 2288 (1987)], Eq. (8) will be derived directly from (3) and the asymptotics will be discussed in more detail. The results are in disagreement with those of Ref. 8.

${ }^{16}$ When plotted on a log-log scale, the data of Fig. 5 of Ref. 7 also yield an effective exponent of about $\frac{1}{2}$ in this range. 